\title{
Improving Local Electrode Performance by Tesla Coil Electric Discharges
}

\author{
D. Isheim ${ }^{1,2}$, A. Akey $^{3}$, and S.S.A. Gerstl ${ }^{4}$ \\ ${ }^{1}$ Department of Materials Science and Engineering, Northwestern University, Evanston, IL, USA \\ ${ }^{2}$ Center for Atom-Probe Tomography (NUCAPT), Northwestern University, Evanston, IL, USA \\ ${ }^{3 .}$ Center for Nanoscale Systems, Harvard University, Cambridge, MA, USA \\ ${ }^{4}$ Scientific Center for Optical and Electron Microscopy (ScopeM), ETH Zurich, Zurich, Switzerland
}

A Tesla coil generates high-voltage high-frequency low-current electric arcing [1]. We have employed a commercially available small hand-held Tesla coil to successfully improve or restore the performance of local electrodes (LEs) used in atom-probe tomography (APT [2]). The local-electrode atom-probe (LEAP) design [3] is advantageous in terms of specimen throughput and flexibility with specimen preparation [3]. The LEs, however, deteriorate with usage, resulting in excessive noise from electron field emission that limits data quality, voltage range, and the field-of-view accessible for APT analysis. Replacing a LE can carry significant cost and efforts have been undertaken to understand failure modes [4] and restoring LEs, e.g. by plasma cleaning [5].

The breakdown voltage of a LE in vacuum can be tested easily in a LEAP with the so-called "flat-test" where a polished clean conductive flat surface is placed opposite to the electrode $[5,6]$ and the voltage increased until electrical breakdown by electron field emission occurs. We placed the flat surface at $\sim 100 \mu \mathrm{m}$ or half the distance to where the flat substrate of a microtip array sample would be located, increasing the electric field by approximately a factor of 2 (or 4 for needle shaped so-called "wire tips") and causing electron emission from the LE to begin at about 50\% relative to the voltage for breakdown during a regular APT analysis. We categorized LE performance determined with the flat test in terms of electron emission beginning at $\sim 5 \mathrm{kV}$ ("usable"), above $7 \mathrm{kV}$ ("good") or above $11 \mathrm{kV}$ ("like new") in reference to voltage limits of $10 \mathrm{kV}, 14 \mathrm{kV}$, or better for APT analyses with microtip array specimens.

We tested LE performance and restoration with the Tesla coil in a multi-user laboratory environment with many different types of samples, including metallic, ceramic, and semiconducting materials, nanowires and nanoparticles, and biominerals, providing a wide-ranging test bed for LE restoration after deterioration from general usage. To restore a deteriorated LE, we activated the Tesla coil by arcing against the surface of a block holding the LEs, Fig. 1, in air, and subsequently directed the arc to the active part (top cone) of the LEs, hitting each LE for 5-10 seconds directly with the discharge. Longer exposure was not found to yield any further improvements. Using the breakdown voltage of air, $>3$ $\mathrm{kV} / \mathrm{mm}$ [7], we estimated applied voltages of about $15-50 \mathrm{kV}$ from the length of the arcs, 5-20 mm. Ten different local electrodes were treated with the Tesla coil during a four-month period, several multiple times each after deterioration from APT use, and after Tesla-coil restoration the flat-test onset for vacuum electron emission determined. The resulting measured improvement of the vacuum breakdown voltage is displayed in Fig. 2, with an average improvement of $(4.5 \pm 1.8) \mathrm{kV}$, and some LEs restored to a "like new" condition by an increase of the vacuum breakdown voltage by about 7-8 kV to above $11 \mathrm{kV}$. SEM imaging confirmed that no melting or damage to the LEs on a meso- or larger scale occurred.

Directing the arc to a contact electrically connected to the LE that exposed them to the voltage applied but not the arc itself did not result in significant improvements. The discharges from the Tesla coil need to hit the active part of the LE directly. Like for the vacuum breakdown in the LEAP, the electric field is 
concentrated at the micro-asperities and this is where the arc from the Tesla coil is most likely to hit the surface as well. Therefore, a possible mechanism for LE improvement could be local submicrometerscale melting of these asperities, due to the high-frequency electric currents and the hot plasma column of the electric arc, possibly in combination with the mechanical impact of the arc or its collapse at the end of a discharge pulse, removing or flattening out of the asperities that cause the vacuum breakdown by electron field emission in the LEAP. Disintegration of micro-asperities by Coulomb forces directly resulting from a surface charge from the electric field does not seem to be a significant process.

In conclusion, we presented a fast and easy to perform process to restore local electrode performance for LEAP atom probes by exposure to electric discharges from a Tesla coil, at a fraction of the cost for a single replacement LE or a plasma cleaner. Better local electrodes allow for analyzing APT specimens with a larger field-of-view, larger datasets with less noise and better overall data quality.

\section{References:}

[1] PH Plesch "High Vacuum Techniques for Chemical Syntheses and Measurements" (Cambridge University Press, Cambridge) 2005, p. 21.

[2] B Gault et al, "Atom Probe Microscopy" (Springer, New York) 2012.

[3] DJ Larson et al, "Local Electrode Atom Probe Tomography: A User's Guide”, (Springer, New York) 2014.

[4] KF Russell et al, Ultramicroscopy 107 (2007), p. 750.

[5] RM Ulfig et al, Microscopy and Microanalysis 15 (2009), p. 286CD.

[6] RV Latham "High Voltage Vacuum Insulation” (Academic Press, London) 1995, p. 20.

[7] JS Rigden, "Macmillan Encyclopedia of Physics" (Simon \& Schuster, New York) 1996, p. 353

[8] The LEAP 4000X Si atom-probe tomograph at NUCAPT (Northwestern University) was acquired and upgraded with equipment grants from the MRI program (NSF DMR-0420532) and the DURIP program of the Office of Naval Research (N00014-0400798, N00014-0610539, N00014-0910781). This work made use of the EPIC facility of the NUANCE Center at Northwestern University. NUCAPT and NUANCE received support from the Soft and Hybrid Nanotechnology Experimental (SHyNE) Resource (NSF NNCI-1542205) and the MRSEC program (NSF DMR-1121262) through Northwestern's Materials Research Center. NUANCE received support from the International Institute for Nanotechnology (IIN); the Keck Foundation; and the State of Illinois, through the IIN. Part of this work was performed at the Center for Nanoscale Systems (CNS) at Harvard University, supported by NSF NNCI-1541959.

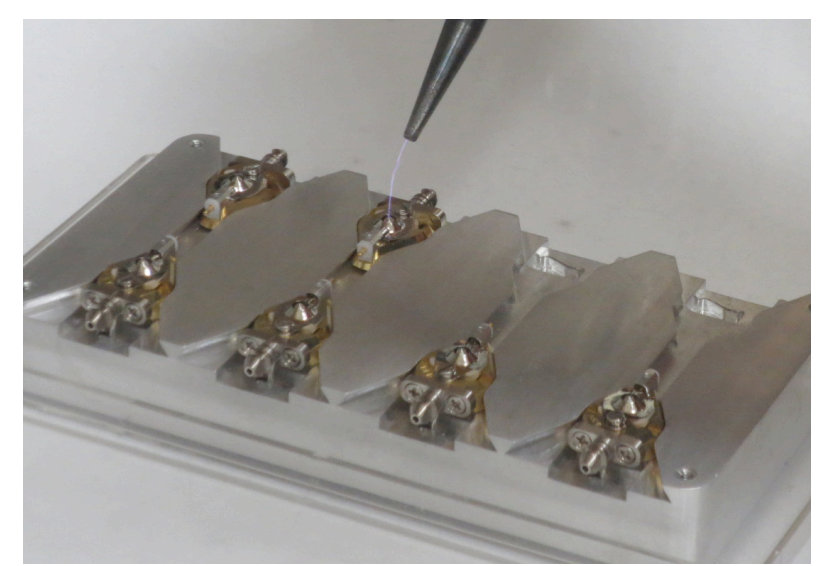

Figure 1. Restoring local electrode performance by applying electric discharges from a hand-held Tesla coil. Photo credit: Stoichko Antonov.

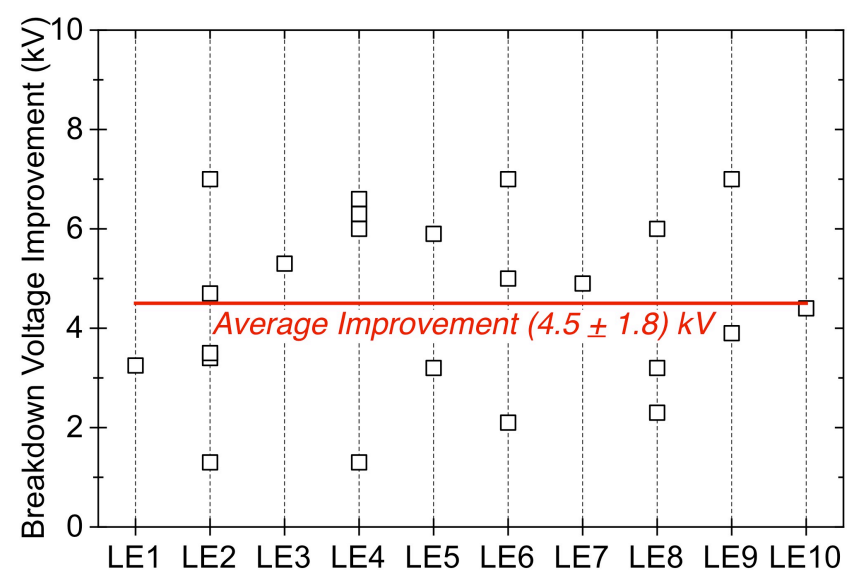

Figure 2. Improvement in local electrode breakdown voltage after treatment with electric discharges from a Tesla coil as shown in Fig. 1. 